\title{
Solar Orbiter's encounter with the tail of comet C/2019 Y4 (ATLAS): magnetic field draping and cometary pick-up ion waves
}

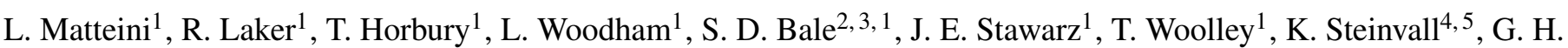
Jones $^{6,7}$, S. R. Grant ${ }^{6,7}$, Q. Afghan ${ }^{6,7}$, M. Galand ${ }^{1}$, H. O'Brien ${ }^{1}$, V. Evans ${ }^{1}$, V. Angelini ${ }^{1}$, M. Maksimovic ${ }^{8}$, T. Chust ${ }^{9}$, Y. Khotyaintsev ${ }^{4}$, V. Krasnoselskikh ${ }^{10}$, M. Kretzschmar ${ }^{10,11}$, E. Lorfèvre ${ }^{12}$, D. Plettemeier ${ }^{13}$, J. Souček ${ }^{14}$, M. Steller ${ }^{15}$, Š. Štverák ${ }^{14,16}$, P. Trávníček ${ }^{3,16}$, A. Vaivads ${ }^{4,17}$, A. Vecchio ${ }^{8,18}$, R. F. Wimmer-Schweingruber ${ }^{19}$, G. C. Ho $^{20}$, R. Gómez-Herrero ${ }^{21}$, J. Rodríguez-Pacheco ${ }^{21}$, P. Louarn ${ }^{22}$, A. Fedorov ${ }^{22}$, C. J. Owen ${ }^{6}$, R. Bruno ${ }^{23}$, S. Livi2 ${ }^{24}$, I. Zouganelis $^{25}$, and D. Müller ${ }^{26}$

(Affiliations can be found after the references)

\begin{abstract}
Context. Solar Orbiter is expected to have flown close to the tail of comet C/2019 Y4 (ATLAS) during the spacecraft's first perihelion in June 2020. Models predict a possible crossing of the comet tails by the spacecraft at a distance from the Sun of approximately 0.5 AU.

Aims. This study is aimed at identifying possible signatures of the interaction of the solar wind plasma with material released by comet ATLAS, including the detection of draped magnetic field as well as the presence of cometary pick-up ions and of ion-scale waves excited by associated instabilities. This encounter provides us with the first opportunity of addressing such dynamics in the inner Heliosphere and improving our understanding of the plasma interaction between comets and the solar wind.

Methods. We analysed data from all in situ instruments on board Solar Orbiter and compared their independent measurements in order to identify and characterize the nature of structures and waves observed in the plasma when the encounter was predicted.

Results. We identified a magnetic field structure observed at the start of 4 June, associated with a full magnetic reversal, a local deceleration of the flow and large plasma density, and enhanced dust and energetic ions events. The cross-comparison of all these observations support a possible cometary origin for this structure and suggests the presence of magnetic field draping around some low-field and high-density object. Inside and around this large scale structure, several ion-scale wave-forms are detected that are consistent with small-scale waves and structures generated by cometary pick-up ion instabilities.

Conclusions. Solar Orbiter measurements are consistent with the crossing through a magnetic and plasma structure of cometary origin embedded in the ambient solar wind. We suggest that this corresponds to the magnetotail of one of the fragments of comet ATLAS or to a portion of the tail that was previously disconnected and advected past the spacecraft by the solar wind.
\end{abstract}

Key words. solar wind - comets - plasmas - waves - instabilities

\section{Introduction}

Serendipitous ion tail crossings can provide valuable information on the structure of a comet's induced magnetotail at great distances downstream of their source regions (e.g. Jones et al. 2000; Gloeckler et al. 2004; Neugebauer et al. 2007). In particular, Jones et al. (2020) reported the near-alignment between the position of Comet C/2019 Y4 (ATLAS) and Solar Orbiter (Müller et al. 2020) in late May-early June 2020, noting that given favourable comet activity levels and solar wind conditions, the spacecraft's in situ instruments would have been able to detect the presence of the comet's ion tail in the solar wind. They suggested that the tail's presence could be evidenced by pickup ions in Solar Wind Plasma Analyzer, SWA data, or the presence of draping signatures in the heliospheric magnetic field by the magnetometer (MAG). Moreover, the interaction of pickup ions with the solar wind can lead to the generation of different types of ion-scale plasma waves that can be measured in situ (e.g. Coates 2004; Ip 2004).

Comet ATLAS reached its perihelion (0.253 AU) in May 2020, after some fragmentation of its main nucleus was observed in March-April 2020. The comet's water production rate was approximately $5 \times 10^{27}$ molecules per second when last observed by the SOHO SWAN instrument, ten days before perihelion (Combi et al. 2021). Despite the fact that it occurred very early in the mission timeline and during the commissioning of the payload, the uniqueness of the event motivated a specific campaign of the Solar Orbiter in situ instruments. This was exceptionally scheduled before the official start of the Cruise Phase described in the Science Activity Plan (Zouganelis et al. 2020), in coordination with the relevant instrument teams and the mission and science operations centres of the European Space Agency. The encounter is expected to have taken place close to Solar Orbiter's first perihelion, at a distance from the Sun of about 0.5AU.

In situ signatures of cometary tail crossings can display different characteristics and shapes depending on the geometry of the encounter, the activity of the comet, the orientation of the interplanetary magnetic field (IMF), and the distance from the Sun (see e.g. Jones et al.|2018, for a review). However, there are some general features that are expected to characterise also those tail crossings occurring much further down from the nucleus (Jones et al. 2010), such as the presence of a neutral, or plasma, sheet, with a very low magnetic field surrounded by two adjacent regions with oppositely directed field, indicative of draping and constituing the induced magnetotail, analogously for the case of a close-nucleus crossing (Slavin et al. 1986). This configuration is typically accompanied by a plasma density increase and a flow 


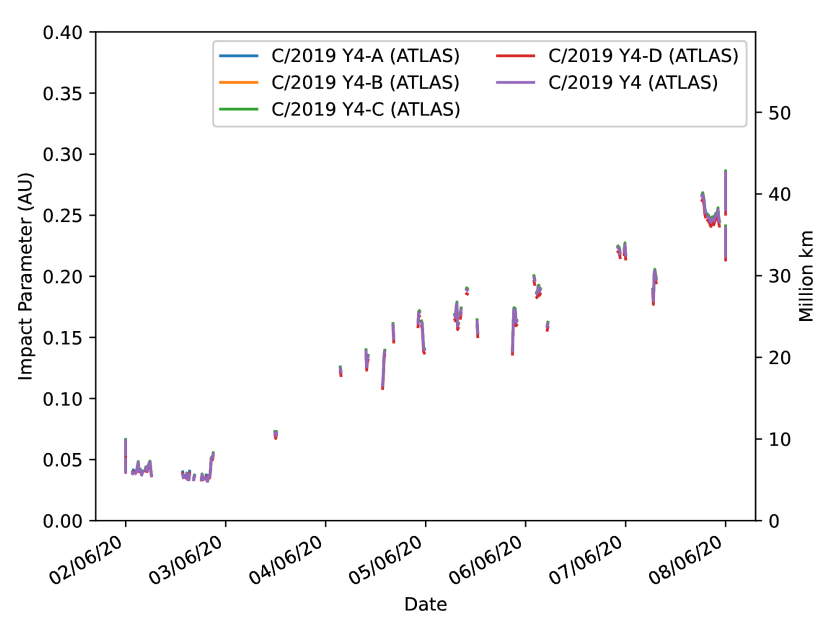

Fig. 1. Impact parameter of solar wind detected by Solar Orbiter as a function of time for the five observed fragments of the parent ATLAS nucleus. Dates are ordered by day-month-year.

deceleration in the tail region, the extended presence of pick-up ions and of intense non-linear waves (e.g. Szegö et al.|2000)

In this work we provide an overview of in situ observations made during the period of the encounter. We first address the large-scale configuration of the plasma, identifying possible signatures of magnetic field draping, then we focus on smaller scale intervals characterized by ion-scale wave activity and potentially related to cometary pick-up ion instabilities. Finally, we reconstruct the geometry of the encounter and discuss possible scenarios for the comet ATLAS magnetotail crossing.

\section{Data}

Here, we exploit data collected by in situ instruments on board Solar Orbiter. Magnetic field measurements cover the whole period, with the exception for a small gap on 3 June, and are provided by the MAG magnetometer (Horbury et al. 2020); magnetic field measurements are shown in RTN coordinates, except in the analysis of small-scale waves and structures, when they are rotated in minimum variance coordinates (MVA). Plasma density measurements were obtained using the Radio Plasma Wave (RPW) spacecraft potential and are available over the full period analysed (Maksimovic et al. 2020, Khotyaintsev et al. 2021). The proton analyser PAS from SWA (Owen et al. 2020) was operating only during the period of 30 May - 1 June. Outside this period, the solar wind speed is estimated using the deHoffmann-Teller analysis of electric field measurements by RPW and magnetic field measurements by MAG (Steinvall et al. 2021). Some statistics for high-frequency waves and dust impact events from RPW/TDS are also used (Píša et al.|2021). Energetic ion fluxes were obtained from Energetic Particle Detector (EPD) STEP measurements (Rodríguez-Pacheco et al. 2020, WimmerSchweingruber et al. 2021).

\section{Comet ATLAS encounter context}

\subsection{Encounter prediction}

Jones et al. (2020) reported that the crossing of ATLAS's ion tail most likely took place around 202031 May - 1 June, based on the assumption of a radial solar wind flow of $300-500 \mathrm{~km} / \mathrm{s}$. Under these conditions, solar wind particles that reached Solar
Orbiter could have approached within $\sim(6.7-7.8) 10^{6} \mathrm{~km}$ from the comet's nucleus. The spacecraft (s/c) crossed the comet's orbital plane on 20206 June. The period around this time was also noted for the possibility that the spacecraft could encounter dust grains of $\beta$ parameter $\sim 1.2$, released from the nucleus on 20 May. This scenario is based on classical modelling of the comet's dust tail (Finson \& Probstein 1968).

The above predictions could be amended by variations from the nominal scenario assumed for the above prediction: a lower solar wind speed would shift the time of closest alignment with the ion tail later. A non-radial wind could also adjust the crossing time either earlier or later, depending on the flow direction. Finally, as Comet ATLAS was known to be fragmenting, it was also known that the source of pickup ions, rather than being an approximately spherical cloud of neutral gas surrounding the nucleus, would instead be an elongated region trailing behind the nominal nucleus position. This too would shift the time of an ion tail crossing later than predicted by Jones et al. (2020).

Using solar wind speed estimates derived from RPW measurements, the actual impact parameter of solar wind detected at Solar Orbiter could be calculated, namely, the minimum distance that each packet of solar wind approached to the comet nominal nucleus position based on radial solar wind flow. We note that the calculation takes into account the aberration caused by the relative speeds between the spacecraft and the comet's orbital motion. The resultant impact parameter is shown in Figure 1 using the ephemeris for each of the five observed fragments of the parent ATLAS nucleus. The data are not continuous, but clearly show that the impact parameter was probably at a minimum on 2 June 2020, reaching at least as low as $5 \times 10^{6} \mathrm{~km}$, and would have been around 15-20 million $\mathrm{km}$ on 4 June. The assumption of an extended ion source from a trail of material would shift the minimum impact parameter period later than indicated.

\subsection{In situ observations}

We can now turn to the analysis of the in situ data for the period in question. The global context of the predicted Solar Orbiter encounter with comet ATLAS is shown in Figure 2. The different panels show: (a-d) magnetic field intensity and components in RTN; (e) RPW plasma density estimated from the spacecraft potential; (f) RPW/TDS statistics of counts of high-frequency waves (black) and dust impacts (red) - counts are accumulated over 2 hours; (g) plasma velocity from SWA/PAS (solid red line) and estimated speed from the RPW electric field (diamonds); (h) number density flux $n V r^{2}$ (in units of $10^{35} \mathrm{~s}^{-1} \mathrm{sr}^{-1}$ ); high energy ion flux from EPD/STEP, the coloured contour indicates fluxes in the range $10^{3}-10^{8}\left[\mathrm{~cm}^{2} \mathrm{ssrMeV}\right]^{-1}$.

Overall, the data reveal the presence of several large-scale structures and polarity changes, associated with transient events (CMEs) as well as HCS crossings, as indicated by labels in the first two panels. Following the time series, we may first note the HCS crossing occurred on May 31st (confirmed by electron pitch-angle measured by EAS, not shown), leading to a sign change in the large scale trend of both $B_{R}$ and $B_{T}$. The HCS crossing is followed by a long period of average negative underlying field polarity when Orbiter was magnetically connected to the Sun's southern hemisphere until another HCS crossing on 7 June, occurring over the course of the arrival of a double-CME (Telloni et al., this issue). Between these main events, a structure associated with some positive polarity was also observed between 3-4 June. The same structure was also observed by Wind and BepiColombo at $1 \mathrm{AU}$, with similar shape and consistent duration (Laker et al.2021), and it is thus interpreted as a corotating 


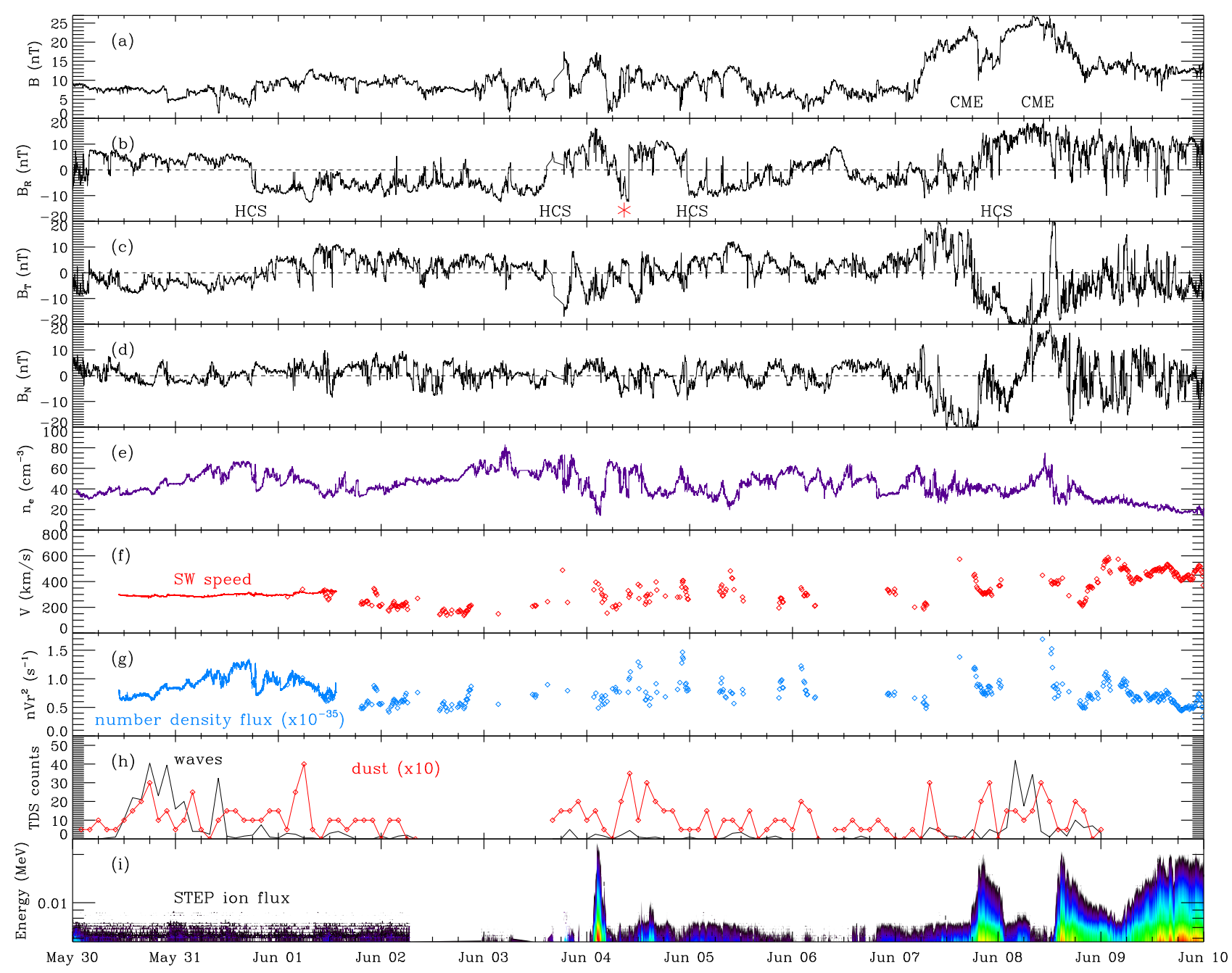

Fig. 2. Context of the Solar Orbiter comet ATLAS encounter. Panels $a-d$ show data from the MAG magnetometer, panel $e$ displays RPW plasma density measurements from the spacecraft potential, while panels $f$ and $g$ show RPW estimations (diamonds) of the flow speed (red) and number density flux (blue). In the same panels, measurements from SWA/PAS are shown as a solid line. Panel $h$ shows statistical counts of high-frequency waves and dust impacts from TDS. The last panel $i$ displays the STEP ion flux; coloured contour refers to flux intensities in the range $10^{3}-$ $10^{8}\left[\mathrm{~cm}^{2} s \mathrm{~s} \mathrm{MeV}\right]^{-1}$. In panel $a-b$, labels indicate HCS crossings localised by Laker et al. (2021) and the double-CME event (discussed by Telloni et al., in this issue). The red asterisk in panel $b$ identifies a magnetic field structure observed at the beginning of 4 June, associated with a full magnetic reversal, a local deceleration of the flow, large density modulations, and enhanced dust and energetic ion events.

structure, that is, likely a ripple in the HCS. Interestingly, inside this positive field structure, over the first half of 4 June, a short interval of reversed field was also observed (red asterisk in panel b), preceded by a region with very low magnetic field intensity; this feature is not seen at $1 \mathrm{AU}$, suggesting that it does not correspond to a corotating structure, and it is the main focus of this work.

Panel e shows the plasma density, inferred from the spacecraft potential. It has enhancements at the HCS crossing, as expected; moreover, it has an absolute minimum on 4 June, just before the magnetic field reversal.

Overall the solar wind speed (panel f) is quite low, a typical condition observed by Orbiter during its early phase and also consistent with close-Sun observation by PSP during the current phase of low solar activity (e.g. Kasper 2019). Although PAS ion measurements cover only the first three days of the encounter, the estimation of the wind speed made using the RPW electric field confirms that the wind speed remains below $400 \mathrm{~km} / \mathrm{s}$ all along the main period, with several subintervals where the speed drops below $300 \mathrm{~km} / \mathrm{s}$. Occasionally, the velocity is probably anomalously low in these subintervals $(V<200 \mathrm{~km} / \mathrm{s})$ but, qualitatively, the data show a clear speed reduction. The measurements also indicate the presence of a faster stream at the end of the interval after the last HCS crossing: this is consistent with the increased level of fluctuations in the magnetic field components, but little variation in the field intensity (panel a-d), as well as a lower and less variable density profile.

Panel $g$ displays the electron number density flux, $n V r^{2}$. Overall, the flux is quite stable, with a similar value for both faster and slower wind streams, as expected; this is a further confirmation that the RPW speed estimation correctly captures the large scale speed variations and, thus, the density flux. Moreover, a value of $\sim 0.5 \times 10^{35} \mathrm{~s}^{-1} \mathrm{sr}^{-1}$ is typical of Alfvénic wind, regardless of its speed (Stansby et al. 2019) and consistent with a typical mass flux of $2-4 \times 10^{8} \mathrm{~cm}^{-2} \mathrm{~s}^{-1}$ measured at $1 \mathrm{AU}$ (Wang 2010). In Figure 2, enhancements in the number density flux above $1 \times 10^{35} \mathrm{~s}^{-1} \mathrm{sr}^{-1}$ are observed at transients and local cross- 
ings of the HCS; remarkably, an additional increase is observed also on 4 June 4 .

Panel h shows some statistics from TDS corresponding to electrostatic small-scale variations that trigger the automatic sampling algorithm by the detector that are typically related to high-frequency waves (Langmuir) and dust impacts. Some enhancements in the wave counts (black) are observed at the beginning and at the end of the interval. A more variable pattern is observed for the dust counts (red), although this can be due to the lower statistics; in particular, the dust counts display an increase around the same time of the magnetic structure of 4 June.

The last panel (i) shows the modulation of suprathermal ions as measured by STEP in the sunward direction. We note that STEP cannot distinguish between different ion species. The flux enhancements seen in this panel can thus be due to protons at their nominal energy or due to heavier ions with the same kinetic energy but correspondingly lower speeds. Depending on the velocity distribution function of the heavy ions and the direction of the magnetic field, this contribution can be substantial. The large increase in the flux at the end of the interval is consistent with the passage to a faster solar wind stream; some modulation associated with the double flux-rope event is also observed. The isolated energetic ion signature at the beginning of 4 June is, on the other hand, more unusual, as it is not associated with significant speed enhancements and could potentially be related to the presence of heavier ions in the plasma. This event covers the full STEP energy range; in addition, there is a small enhancement also seen at 60-70 keV in the EPD/EPT sensor (not shown), which looks in the same direction as STEP.

All these features suggest that the isolated structure observed on 4 June could be related to a transient event and because of the vicinity to the expected encounter with comet ATLAS, in the next section, we further investigate whether these signatures could be caused by the interaction of the solar wind plasma with some cometary material. Moreover, this period is also rich in ionscale wave-forms whose shape and properties are unusual in the typical solar wind. This latter aspect is investigated in Section 5 .

\section{Analysis of 4 June}

\subsection{In situ data}

In this section, we focus on measurements from 4 June, as shown in Figure 3 . In particular, we address in greater detail the substructure of the field observed during the first half of the day. We consider first the interval between 00:00 and 12:00 in Figure 3, where the sub-intervals are indicated by labels A-B-C-D and separated by vertical thin dotted lines. This interval is characterised by the presence of an extremely low magnetic field region (B) observed between 4:00 and 8:00 (with a field intensity drop down to $B \sim 2 \mathrm{nT}$, seen in the top panel). This region is bounded by oppositely directed field (regions $\mathrm{A}$ and C): $B_{R}$ reverses completely (panel c), and, similarly, $B_{T}$ and $B_{N}$ (panel d). Magnetic field intensity variations are almost perfectly followed by anti-correlated electron density changes (red line, panel b); although there are no temperature measurements available for this interval, the density behaviour suggests that the plasma is likely very close to pressure balance. Region $\mathrm{C}$, with a reversed field seen approximately between 8:00 and 10:00, ends with a sharp rotation of $\mathbf{B}$, very clear in the cone-angle $\theta_{B R}$ (panel g), just before 10:00. This discontinuity separates two regions of plasma with different density (almost a factor 2). After the density jump, in region $\mathrm{D}$ the magnetic field switches back to the orientation and amplitude observed at the beginning of the day (before 4:00), corresponding to a background positive polarity (with negative $B_{T}$ ). However, the density remains significantly higher than the beginning of the interval, thus suggesting some local plasma compression instead of pressure balance.

Figure 4, shows in detail the discontinuity occurring between 9:47 and 9:50, and separating the two plasma regions C and D discussed above. The magnetic field intensity (cyan, top panel) drops to almost 0 inside the structure. The magnetic field components are presented in minimum variance coordinates and display the typical properties of a current sheet with almost antiparallel field at the two sides (black line), with a very small guide field (green line). The third component (orange), is close to zero everywhere, except for some variations inside the sheet. These are highlighted by the bottom panel, showing the magnetic field cone angle $\theta_{B R}$, and indicative of some sub-structure inside the current layer. Finally, the top panel shows also the density (red line), which jumps from $30 \mathrm{~cm}^{-3}$ to $60 \mathrm{~cm}^{-3}$ across the discontinuity.

The other panels in Figure 3 further characterise the plasma configuration around this magnetic structure. Panel e displays the wind speed $V$ estimated by RPW; there is a clear flow deceleration between the first two regions, with a very slow solar wind in region $\mathrm{B}$. While, occasionally, some values of the speed could be anomalously low, the systematic decrease indicates that the solar wind speed is reduced in region B, while the speed increases again in region $\mathrm{D}$. There are no reliable measurements of the speed in region $\mathrm{C}$. The following panel (f), shows the number density flux $n V r^{2}$; despite the deceleration between regions $\mathrm{A}$ and $\mathrm{B}$, the density flux remains quite constant through them (probably a consequence of the pressure balance), while there is a signature of enhanced density/mass flux in region $\mathrm{D}$, suggesting a possible local accumulation of the plasma, responsible for some compression and loss of the pressure balance condition (first panel).

Panel h shows the EPD/STEP measurements of suprathermal ions for 3 energy channels covering energy deposition between 6-19 keV. Interestingly, the peak already seen in Figure 2 and previously discussed is constrained to region A; moreover, its modulation is correlated with the variations in $V$. Since the magnetic field does not change its direction significantly in region $\mathrm{A}$ and until after inside region $\mathrm{B}$ (see panel $\mathrm{g}$ with $\theta_{B R}$ ), changes in the STEP flux are not due to field of view variations and it is reasonable to assume that the observed flux modulation is associated with the local variations in the speed. This has two important consequences: first it is a benchmark of the RPW speed estimation; second, although STEP cannot identify ion masses, the signature would be consistent with it being generated by heavier ions whose energy is modulated following $V$. For example, $O^{+}$ cometary ions picked-up at a speed close to the solar wind velocity would be detected by STEP in region A $(V \sim 400 \mathrm{~km} / \mathrm{s})$ but not in region B, where $V$ drops to $\sim 200 \mathrm{~km} / \mathrm{s}$ because heavy ions would not have enough energy to penetrate the STEP detector's deadlayer and deposit sufficient energy to trigger the detector.

To better appreciate the changes in the $\mathbf{B}$ orientation during the whole crossing described, a projection of the magnetic field vector in the RT plane is shown in the bottom panel (i) of Figure 3 We note the slow full rotation of the field around the lowfield region $\mathrm{B}$ and the quick reversal at the current sheet crossing between $\mathrm{C}$ and $\mathrm{D}$. Another reversal occurs at the end of the day, due to the crossing of the HCS (see also Figure 2). 

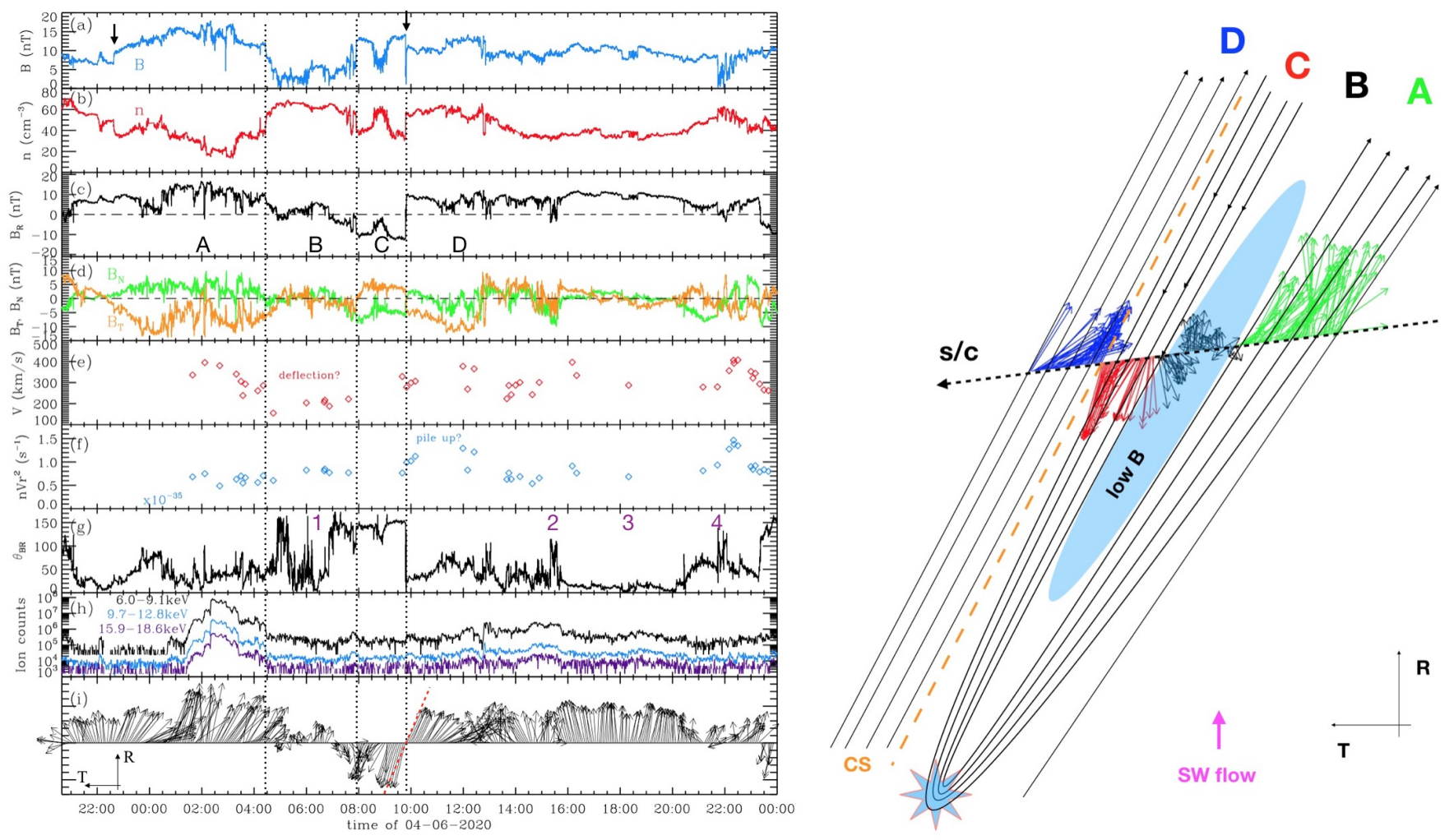

Fig. 3. Solar Orbiter crossing of the ion tail of comet ATLAS. Left: Overview of the ion-tail encounter starting at the end of 3 June until the end of 4 June. Panel a shows magnetic field intensity $B$ (blue) and panel $\mathrm{b}$ the plasma density $n$ (red). Panels $\mathrm{c}$ and display the magnetic field components. Labels in panel $\mathrm{c}$ refer to the four regions A-B-C-D identified in the text and separated by vertical dotted lines. Panels e and $\mathrm{f}$ show the estimated RPW wind speed $V$ and the associated number density flux, respectively. Panel g shows the magnetic field cone angle $\theta_{B R}$ and numbered labels indicate intervals of wave activity analysed in Section 5 Panel h contains STEP ion counts for three selected energy channels in the range of 6-19keV. The last panel, labelled i, shows a time series of the projection of the magnetic field vector in the plane RT; the length of the arrows is proportional to the intensity of the field in the plane. The dashed red line shows the orientation of the discontinuity between regions $\mathrm{C}$ and $\mathrm{D}$ resulting from the MVA analysis. The arrows in the top panel indicate the boundaries of the A-B-C structure, as discussed in Section 6 Right: Diagram of the reconstruction of the magnetic field structure observed in the A-B-C-D regions. The magnetic field vectors of panel $\mathrm{h}$ are plotted on top of an approximate Solar Orbiter trajectory through the figure. The dashed orange line encodes the current sheet (CS) of Figure 4 and its orientation corresponds to that of the red dashed line in panel $\mathrm{i}$.

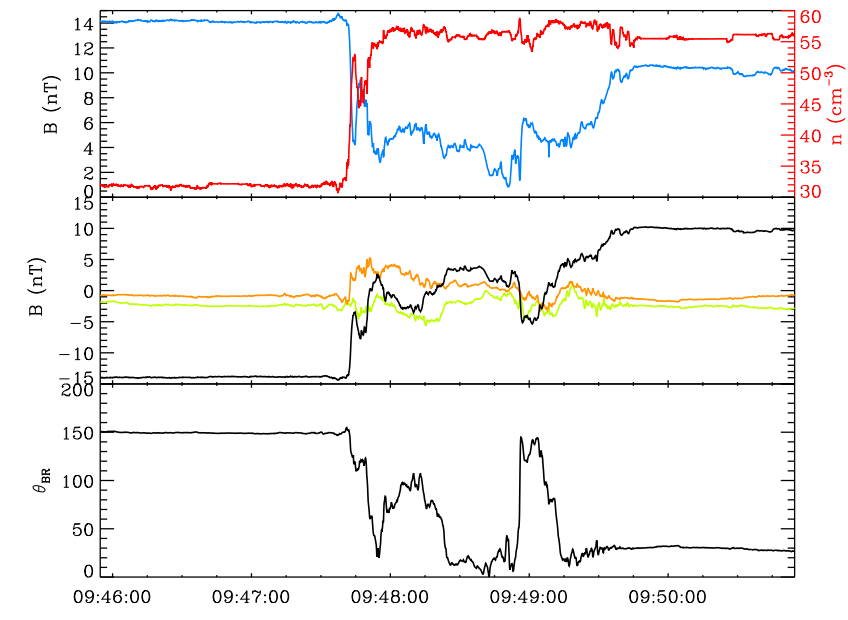

Fig. 4. Small-scale sharp current sheet observed on 4 June, separating plasma regions $\mathrm{C}$ and $\mathrm{D}$ with anti-parallel $\mathrm{B}$-field and with different electron density. Top panel shows the total magnetic field (blue), which drops to almost zero inside the sheet, and the electron density (red). The middle panel displays the magnetic field components in MVA, while the bottom panel shows the angle $\theta_{B R}$ between the magnetic field and the radial.

\subsection{Encounter reconstruction}

We go on to discuss the origin of the whole region observed. As mentioned, it is embedded in a positive polarity interval associated with a small corotating region, which is stable enough to be observed also at $1 \mathrm{AU}$ (Laker et al. 2021) and can be interpreted as a ripple in the HCS that crossed in and out on 3 June and at the end of 4 June, respectively. If the brief negative polarity interval between 8:00 and 9:50 (region C) was due to another smaller ripple structure of the HCS, that would require another double crossing of the HCS at the two boundaries. The second of those ought to correspond to the discontinuity between regions $\mathrm{C}$ and D, as shown in Figure 4, this is a very clean and sharp short structure that is unlikely consistent with a proper HCS crossing. Moreover, this field reversal is not observed at larger distances, suggesting that this is not a corotating structure.

A more likely alternative scenario assumes that the brief change of polarity observed in region $\mathrm{C}$ is not related to a different polarity source at the Sun and, instead, that it is associated with comet ATLAS, as suggested by many of the independent properties discussed above. Our interpretation is that the reversed field corresponds to some draping around the highdensity and low-field region. A sketch of the magnetic field draping around region B is shown in the right panel of Figure 3 . The diagram is in the average RT plane of the encounter and provides 

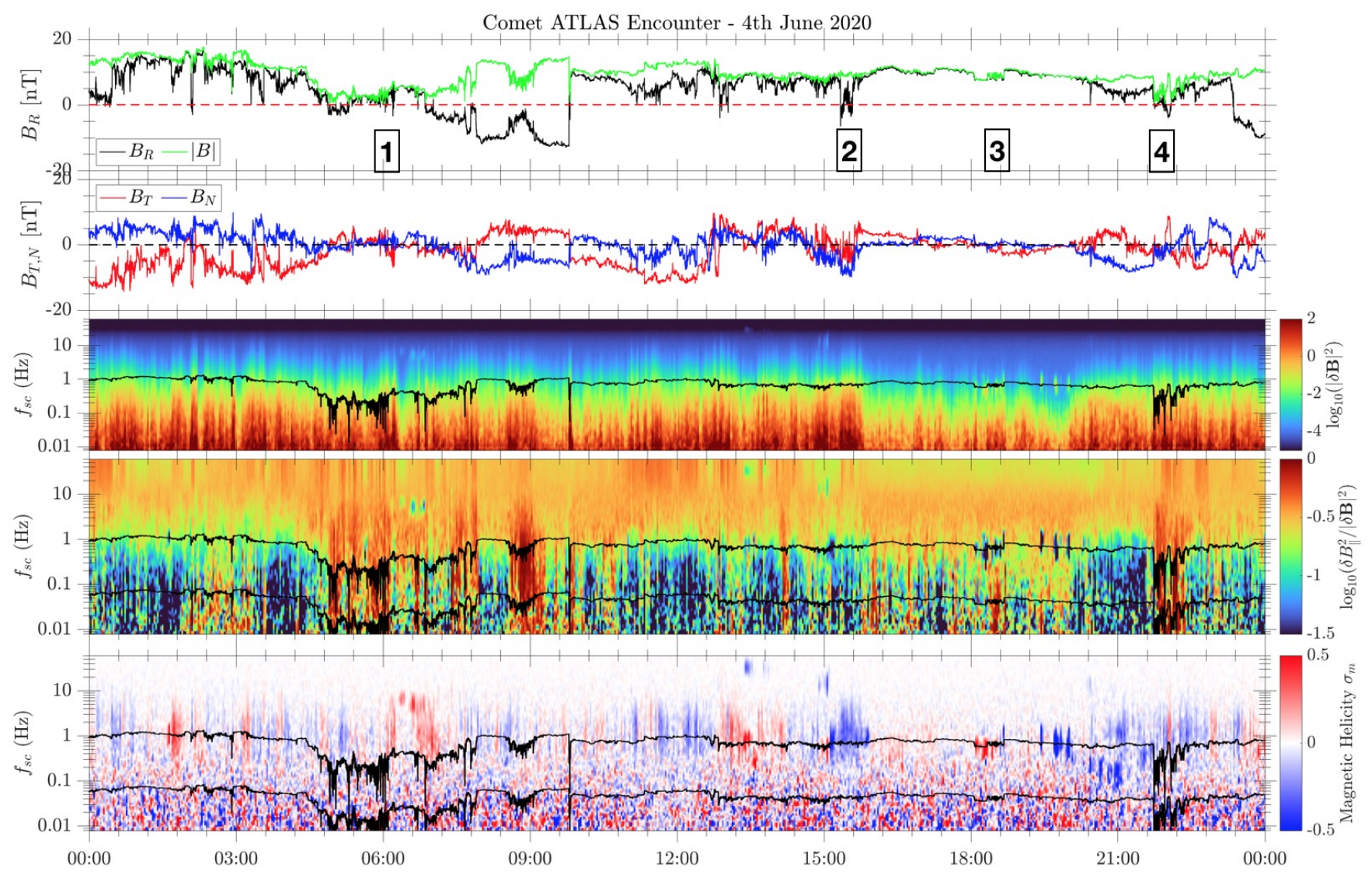

Fig. 5. Overview of wave properties on 4 June. First and second panel show magnetic field intensity $B$ and RTN components. The other panels show spectrograms of the power, magnetic compressibility and magnetic helicity of the fluctuations, respectively. Solid black lines indicate the proton (around $1 \mathrm{~Hz}$ ) and Oxygen $O^{+}$gyrofrequencies. Numbered labels in the top panel indicate the intervals addressed in the paper.

the reconstruction of the magnetic field configuration around regions A-B-C-D; field lines in regions $\mathrm{A}$ and $\mathrm{C}$ are drawn as connected, like for a field draped around an obstacle placed closer to the Sun. We note that the sketch is not to scale since, in the reconstruction, the magnetic field kink is caused by comet ATLAS at 0.25 AU, while Solar Orbiter samples plasma at 0.5 AU. The solar wind comes from below and the dashed black line encodes the spacecraft trajectory through the advected plasma (assumed to be mostly transverse to the structure since the $\mathrm{s} / \mathrm{c}$ motion is dominated by the tangential velocity in this interval, $\left.V_{T}^{s / c} \sim 40 \mathrm{~km} / \mathrm{s}\right)$. Along this trajectory, we report the magnetic field vectors the same way as in the bottom panel of Figure 3 . using different colours for the four sub-regions. We note that the vectors are shown in the same reference frame in the two plots (RT plane), so their orientation is the same both in the time series of panel $\mathrm{h}$ and in the cartoon diagram. The discontinuity between regions $\mathrm{C}$ and $\mathrm{D}$ is indicated by the dashed orange line (CS).

This reconstruction takes into account all measurements in the left panel of Figure 3 and is consistent with all the observations previously discussed. In region A, the magnetic field has the orientation of the background Parker spiral and we observe a gradual deceleration of the flow speed. This is then connected to region $\mathrm{B}$, which is the core of the structure, with low field and high electron density; this is also where the flow is the slowest, as the plasma sheet of the comet magnetotail. Beyond it, we find region $\mathrm{C}$, where the field lines are reversed; this region is the consequence of the draping. In all these regions, the plasma is in pressure balance; this configuration ends with a discontinuity - a sharp anti-parallel current sheet - that bounds region $\mathrm{C}$ and sep- arates it from region $\mathrm{D}$, where the magnetic field is back to the ambient orientation and the solar wind is faster. By applying a minimum variance analysis (MVA) to the discontinuity between regions $\mathrm{C}$ and $\mathrm{D}$, it is possible to estimate the orientation of this boundary of the tail. This is shown with a dashed red line in the panel $i$ of Figure 3 , the resulting orientation is very close to that of the underlying interplanetary magnetic field and the same angle is shown for the dashed orange line in the cartoon reconstruction. The MVA analysis is then consistent with the comet tail configuration in the sketch and suggests a moderately nonradial orientation towards the $-\mathrm{T}$ direction. Such a $-\mathrm{T}$ deflection of the ion tail agrees with expectations based on comet ATLAS's prograde orbital motion.

\section{Waves and structures on 4 June}

In this section, we address in greater detail some selected intervals on 4 June where ion-scale waves have been identified. Figure 5 shows an overview of the fluctuations observed over the whole day. The first two panels show the time profile of mangetic field intensity, $B$, and of the components. The third panel displays a spectrogram of the power of the fluctuations as a function of time and frequency in the spacecraft frame; the solid black line encodes the local proton cyclotron frequency, as a reference. The fourth and fifth panels show the spectrogram of the magnetic compressibility (e.g. Matteini et al. 2020) and reduced helicity (e.g. Woodham et al.2018), respectively; in these panels, the upper and lower solid black lines encode the proton and Oxygen $O^{+}$cyclotron frequencies, respectively. We can see 
that overall, there are signatures of circular polarization waves (distinct non-zero magnetic helicity) around the proton scale in several intervals and that the compressibility of the plasma is enhanced in regions where $B$ is lower. Also, as expected, the magnetic compressibility is lower (small $\delta B_{\|}$) in correspondence of coherent packets with large helicity (i.e. transverse waves). It is worth noting that above $10 \mathrm{~Hz}$, the signal is dominated by the instrumental noise floor.

In the rest of this section, unless otherwise stated, all plots are in MVA coordinates and magnetic field components for maximum, intermediate, and minimum variance are indicated as: $B_{\text {max }}, B_{\text {int }}, B_{\text {min }}$, respectively. In all panels, we use the following colour convention: density, $n$, in red; magnetic field intensity, $B$, in blue; $B_{\text {max }}, B_{\text {int }}$, and $B_{\text {min }}$ in black, orange, and green, respectively. Intervals shown and discussed below are indicated by numbers in the top panel of Figure 5, for reference, also see panel $g$ of Figure 3 .

\subsection{Train of arc-polarised waves - interval 1}

We first considered an interval that is located well inside the region B of Figure 3 (low $B$ ); this is indicated by label 1 in Figure 5 Between 4:00 and 8:00, various types of fluctuations and wave packets were observed, whose shapes are not commonly seen in the typical solar wind, including some waves with very steepened fronts and sharp rotations of the field. One of the most distinct of these features is shown in the top panel of Figure 6 . this corresponds to a train of rotational discontinuities, lasting approximately five minutes. The structure produces little compression of the total magnetic field and density (blue and red lines, respectively), suggesting an underlying Alfvénic nature. The very large variations in $B_{\max }$ look like steepened Alfvén waves; such structures can be observed in the solar wind (Tsurutani et al. 2018), but are typically more isolated features. The clear periodicity of the waves in Figure 3 is striking and more unusual. Series of steepened Alfvén waves have been identified in cometary environments before (Tsurutani \& Smith 1986), supporting their cometary origin.

A slightly larger interval including this event is shown in the middle panel of Figure 6 . We note that the wave packet is preceded by a similar, but less extended isolated steepened structure at around 6:06. The two packets are separated by a quieter region. Interestingly, the first detected packet displays a steepening in the opposite direction than the second. This is suggestive of an opposite propagation direction in the plasma frame and thus a possible common source between them. Moreover, the structure detected first has a front direction indicative of antisunward propagation and thus consistent with an earlier arrival at the spacecraft, while the steepening of the more periodic packet of the top panel of Figure 6 is consistent with sunward propagation, leading to a later detection. As mentioned above, one possible interpretation is that these waves are generated by cometary pick-up ions.

Another possible interpretation, based on the fact that these waves are observed in region $\mathrm{B}$, where some velocity shear is likely present due to the magnetic field draping, is that they correspond to Kelvin-Helmhotz (KH) vortices. The bottom panel of Figure 6 shows a further zoom out of this interval, revealing that the waves are observed on top of a background magnetic field and density gradient, connecting directly to the region of lowest field $(B \sim 2 n T)$. Unfortunately, the RPW speed estimation does not have a high enough cadence to determine whether a similar gradient occurs also in the underlying flow velocity; we note, however, that due to the low magnetic field, the Alfvén speed is

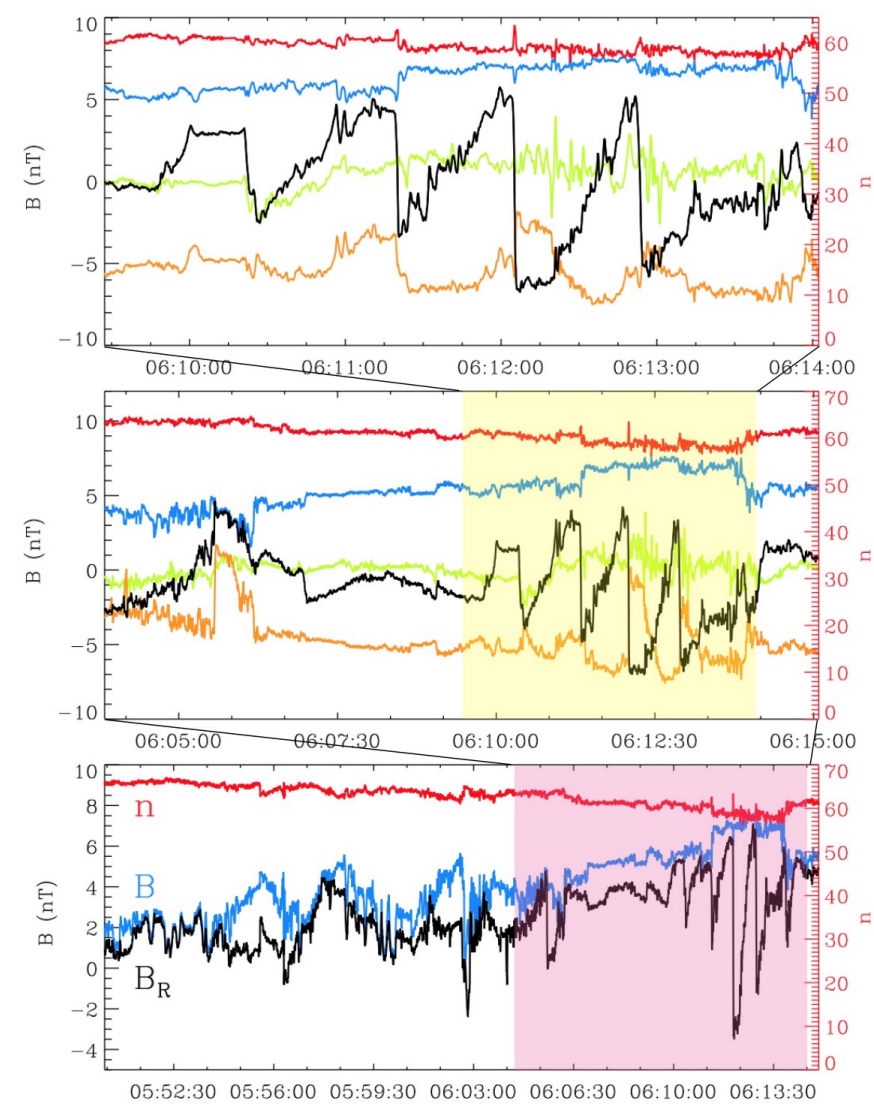

Fig. 6. Interval 1. Top: Train of rotational discontinuities/steepened Alfvén waves observed inside the low field region B (corresponding to interval 1 in panel g of Figure 3 . The red and blue lines encode the density $n$ and magnetic field $B$, respectively, and B-components are shown in MVA $\left(B_{\max }=\right.$ black, $B_{\text {int }}=$ orange,$B_{\min }=$ green $)$. Middle: Larger interval around the event of the top panel, showing the presence of a counterpropagating packet, with wave-front steepened in the opposite direction and detected earlier by the s/c. Bottom: Larger interval containing the wave packets, showing that they are located on a magnetic and density gradient connecting a region of very low field $B$. In this panel, the black line corresponds to $B_{R}$.

also quite low during this period $\left(v_{A} \sim 15 \mathrm{~km} / \mathrm{s}\right)$ and the triggering of a $\mathrm{KH}$ instability is a likely outcome. Some $\mathrm{KH}$ vortices crossed by Solar Orbiter have been detected in the vicinity of the HCS (Kieokaew et al. 2021), but their characterisation implies plasma velocity measurements that are not available for this interval.

While it is probably not possible to discriminate between these two instabilities, we note that both cases are consistent with a cometary origin - pick-up ions or velocity shears at the draping boundary - and fit well with the large scale reconstruction discussed in the previous section.

\subsection{Ion-cyclotron waves - interval 3}

As we can see from Figure 5, an intense ion-scale wave activity is observed also outside of the main cometary region, with many signatures of distinct circular polarization (non-zero magnetic helicity in the bottom panel). Figure 7 shows an example of ion-cyclotron-like waves detected around 18:00, during a period when the magnetic field is very radial and the solar wind is particularly quiet (interval 3). These waves are essentially trans- 

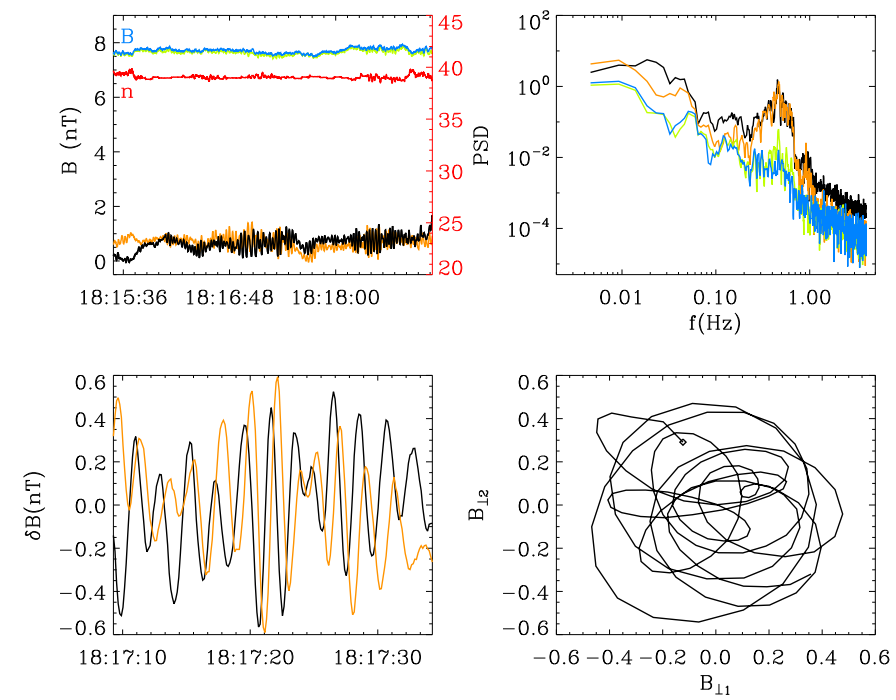

Fig. 7. Example of waveform corresponding to proton scale ioncyclotron waves measured during interval 3 in Figure 5 Magnetic field components and density are shown in top left panel with the same colour code of Figure 6 Top-right panel shows the power spectrum of the fluctuations. The bottom-left panel shows the fluctuating transverse components over a shorter interval, while the bottom-right panel displays is the corresponding hodogram. the diamond identifies the beginning of the interval plotted.

verse and cause little change in the total magnetic field intensity and density(top left panel). They are quasi-monochromatic, with a narrow and large-amplitude peak in the spectrum between 0.1 and $1 \mathrm{~Hz}$ (top right), corresponding to wavelengths around the proton scale (the ion inertial length is $d_{p}=36 \mathrm{~km}$ in this interval, corresponding to an advected frequency $f_{d} \sim 1 \mathrm{~Hz}$ ). The bottom left panel shows a shorter interval, with fluctuations in the transverse components ( $B_{\text {max }}$ and $B_{i n t}$, average removed), displaying a $\pi / 2$ phase difference, corresponding to a well defined circular polarisation also visible in the hodogram (bottom-right panel). The observation of ion-cyclotron waves during quiet radial solar wind periods is not unusual (Jian et al. 2014), especially in the inner Heliosphere (Jian et al. 2010; Verniero et al. 2020); these waves, which can have either a left- or right-handed polarization in the plasma frame, are typically associated with the presence of non-thermal proton distribution functions and likely generated by kinetic instability processes related to ion temperature anisotropy, or field-aligned secondary beams (Woodham et al.2019); at planetary atmospheres and comets, they can also be generated by pick-up ions (e.g. Delva et al. 2008). The generation of ion-cyclotron waves close to the proton scale in this period could then be related to the presence of $\mathrm{H}^{+}$ions from the comet with unstable velocity distributions (Neugebauer et al. 1989). The enhanced peak in the spectrum is suggestive of a nearby strongly unstable source for the waves. However, we cannot exclude the possibility that these waves are part of the background solar wind and are generated by an instability of thermal solar wind protons. Although proton scale waves in cometary environments have been observed before (Mazelle \& Neubauer 1993), we also note that we can expect most of the wave activity around comets to be generated by heavier ions (rather than $\mathrm{H}^{+}$) as it seems to be the case in the interval considered in the next subsection.

\subsection{Transverse linearly polarised waves - interval 2}

Not all the waves observed during this period are circularly polarised and associated with proton scales. Figure 8 shows a waveform with a period of a few tens of seconds and a spectral contribution below $0.1 \mathrm{~Hz}$ and, thus, with a substantially lower frequency (larger convected spatial scale) than the typical ioncyclotron waves just discussed. The top-left panel shows, as in Figure 7 that the waves are mostly transverse, with little perturbation of total $B$ and density. This is confirmed by the power spectrum in the top right panel, where the amplitude of the transverse components (black and orange) is dominant in the lower frequency range. The wave period observed here is on the order of $\sim 30$ s, by way of a comparison with the ion-cyclotron waves discussed in the previous section and with spectral peak at $0.5 \mathrm{~Hz}$, this is approximatively 15 times larger, making it unlikely that these waves are associated with protons. Instead, their origin is more likely associated with heavier ions, for instance, $\mathrm{O}^{+}$released by the comet. This is further supported by the fact that this interval is number 2 in panel $g$ of Figure 3 and we note that it is also associated with some local enhancements in the STEP ion counts.

Interestingly, however, this wave packet does not display the typical circular polarization of the ion-cyclotron modes. Instead, the zoom-in shown in the middle-left panel, reveals a linear polarization of the fluctuations (either 0 or 180 phase between the transverse components, shown in solid and dashed line, respectively). Despite the linear polarisation, the fluctuations remain perpendicular to the ambient magnetic field, so that the wave is not compressive - as demonstrated by the quite steady profiles of $B$ and $n$ in the top left panel. The hodogram of the transverse components, shown in the middle-right panel, sheds light on this behaviour. To guide the reader, each wave period of the short time series shown in the bottom-left panel is indicated with a different colour, so that it is possible to track them along the hodogram. Each of the individual oscillations, identified by a different colour, occurs along an axis in the plane orthogonal to $B$, as expected for linear polarisation. However, the orientation of such an axis is not constant in time, and it precesses around $B$ during the wave motion. This corresponds to a transverse linearly polarised wave whose axis rotates in time around the background magnetic field. The period of this rotation is on the order of $90 \mathrm{~s}$; the proton gyro-period is $6 \mathrm{~s}$ in this interval, so that the axis rotation is very close to the cyclotron period of an Oxygen ion $\mathrm{O}^{+}$. This is, again, suggestive of a possible cometary plasma origin for these waves.

Waves with a similar properties - namely, linear polarisation and precession of the polarisation axis around the magnetic field - have been predicted by numerical simulations (Matteini et al. 2015). This work investigates instabilities generated by cometary pick-up ions in the solar wind, including the case of a non-gyrotropic source, as expected for an unsteady cometary emission. The bottom panels of Figure 8 report the results of Matteini et al. (2015) in the case of an initially unstable distribution of cometary pick-up $O^{+}$ions that is non-gyrotropic; this configuration generates unstable waves along the background magnetic field that scatter the ions bringing the plasma towards a more stable state. In the simulation, $B_{x}$ and $B_{y}$ correspond to the transverse component of the fluctuating magnetic field and their spatial profile in the simulation box at a given time during the wave growth is shown in the bottom-left panel. The waves excited in the plasma are linearly polarised and the instantaneous polarisation axis measured along the simulation box changes over time (bottom-right panel), leading to a hodogram 

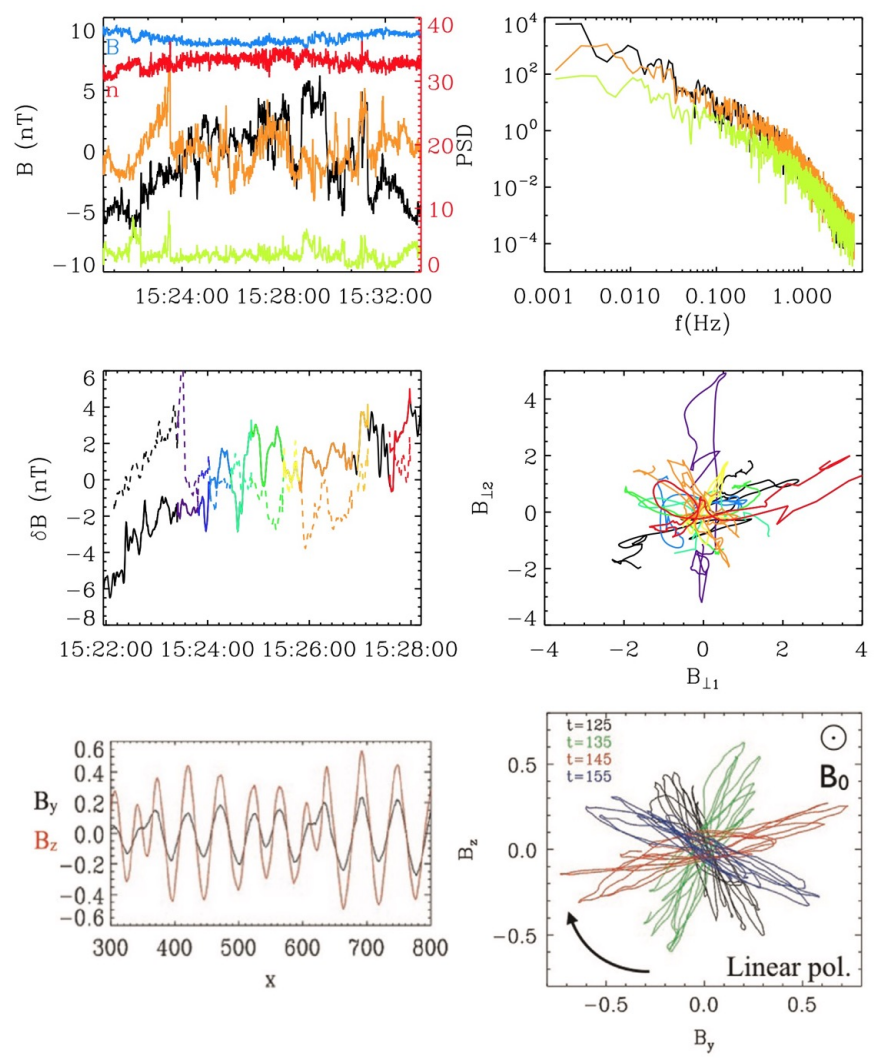

Fig. 8. Interval 2. Top panels: MVA fluctuations and associated power spectra for interval 2 in Figure 5 Middle panel: Solid and dashed lines in the left panel encode the transverse components of the fluctuations; the two components display a linear polarization (signals in phase or anti-phase). This is confirmed by the hodogram in the right panel: different periods of the wave-form are indicated with different colours; each wave period (colour) correspond to a linear polarization axis with a different inclination in the perpendicular plane. Bottom panels: Hybrid simulations results from the instability of non-gyrotropic cometary pick-up ions (from Matteini et al. 2015). As for the middle panels, the bottom left shows the two components of the magnetic field orthogonal to the background field and bottom right their hodogram at different simulation times. The behaviour seen in the simulation matches the observations remarkably well (see text for a discussion).

pattern in the perpendicular plane that is very similar to that of the observations. The explanation of the observed behaviour in the simulations is based on the fact that the cometary ions were not injected initially in the solar wind plasma with a gyrotropic distribution but, rather, with a distribution corresponding to a gyrophase bunch in phase space. As a consequence, while supporting the growth of the unstable waves, they continue to gyrate around the magnetic field, thus causing a rotation of the polarisation axis of the excited waves, which tracks their instantaneous gyrophase and has the same period of their gyration. In the simulation, this occurs at the gyro-period of the unstable $O^{+}$ions and is consistent with the timescale observed in the data.

\subsection{Compressive perpendicular waves - interval 4}

From Figure 3, we notice that towards the end of the day, Solar Orbiter sampled another short region with a significantly low B-field and higher and more variable electron density, between approximately 21:30 and 22:15. The top panel of Figure 9 shows this region in more detail; there are large-scale variations in $B$
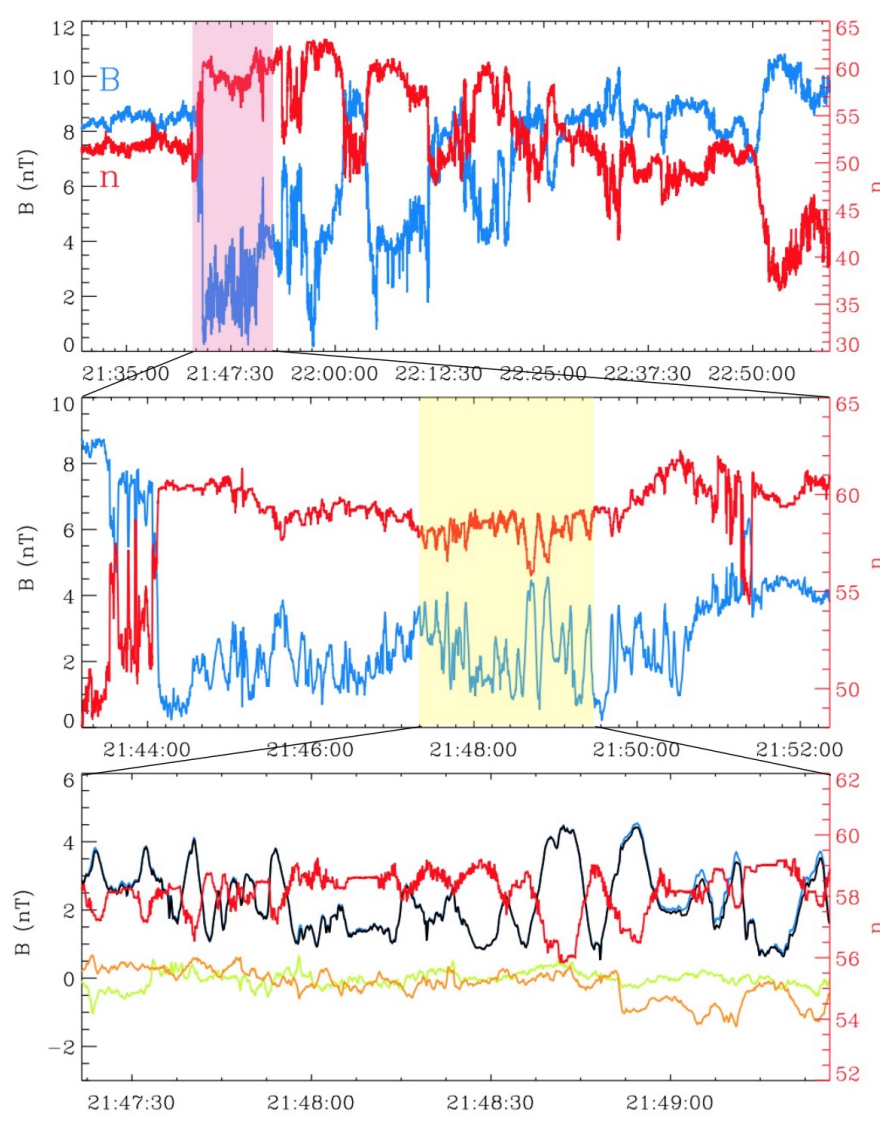

Fig. 9. Top panel shows the decrease in the field intensity $B$ (blue) and the anticorrelated density increase (red) - observed towards the end of the day, before the HCS crossing in a region of highly compressible (interval 4 in panel g of Figure 3). Middle panel shows a detail of the fluctuations located in the region where the field is lowest (light purple region in the top panel). This is characterized by fluctuations in $B$ that are of the same order of the background field. Bottom panel shows a zoom in the compressible structure (yellow region in the middle panel). These ion-scale fluctuations display anti-correlation between $B$ and $n$, while there is little power in the other components $B_{\min }$ and $B_{\text {int }}$; as a consequence the $B_{\max }$ profile overlaps with the field intensity $B$.

and $n$ with large amplitudes and which are anti-correlated in a way that is consistent with pressure balance.

The middle panel of Figure 9 focusses on the fluctuations that are observed in the region where the magnetic field intensity is the lowest. This reveals indeed an ion-scale structure with modulation of a few seconds and with very different properties with respect to Alfvénic perturbations discussed previously. Large variations in both the magnetic field intensity and density are visible, revealing the presence of a highly compressive wave, with $\delta|B| \sim B$. Due to the particularly low $B$ and the high density, the plasma beta is likely large in this interval; this is consistent with fluctuations that are order $\sim 1$, in the magnetic field, but with much smaller relative amplitude, $\delta n / n$.

Unlike other events earlier in the day, when the magnetic field was often aligned with the flow direction, leading to the detection of mostly parallel propagating waves, such as those discussed in previous sections, during the interval shown in the middle panel, the angle between the magnetic field and the radial was close to 90 degrees. This allows us to explore a different region of k-vectors with respect to the previous events and, in particular, to test the presence of waves and structures with a strong perpendicular modulation. The bottom panel is a further 
zoom onto this interval. Magnetic fluctuations are clearly dominated by variations in only one component $\left(B_{\text {max }}\right)$; due to the solenoidal condition for $\mathbf{B}$, these cannot be longitudinal modes and thus, it is implied that there is a very oblique k-vector with respect to the local $\mathbf{B}$, consistent with the sampling direction during this interval. Given the strong anti-correlation between magnetic field intensity and the density, this is likely a nonpropagating and pressure-balanced perpendicular structure, for instance, an ion-scale slow mode. The observed pattern is consistent with an orthogonal s/c cut through a series of field aligned tubes. As the proton inertial length $d_{p}$ is $\sim 10 \mathrm{~km}$ in this interval, and the flow speed is around $300 \mathrm{~km} / \mathrm{s}$, the advected scale of $d_{p}$ is on the order of $10 \mathrm{~s}$, which is comparable to the wavelength of the waveform in bottom panel. The size of the tubes is, thus, on the order of the proton inertial length.

The origin of this periodic structure is not clear. Pressurebalance structures with highly oblique k-vectors can be generated by the mirror instability and are typically observed in planetary magnetosheaths and sometimes in the solar wind as well. More commonly, they are characterised by dips in the magnetic field (and density peaks), however, theory and simulations predict mirror modes with peaks in $B$ (e.g. Califano et al. 2008), which are also found in situ at times (Soucek et al. 2008). The mirror instability requires large perpendicular ion anisotropies and some large ion $\beta$; these conditions are likely met in this low$B$ interval where, as mentioned, the plasma beta is expected to be high, and since pick-up ions have typical large perpendicular temperatures, we have $T_{\perp}>T_{\|}$. On the other hand, such a perpendicular modulation of the magnetic field could also be produced by a filamentation instability (Shukla \& Stenflo 1989, Laveder et al. 2002, Borgogno et al. 2009) induced by pick-up ions. Whatever instability is the case, given the wavelength of the modulation that is closer to proton scales than those of heavier ions, it is not likely that this structure is caused by heavier pickup ions, such as $\mathrm{O}^{+}$; consistently, there are no energetic ions signatures in the STEP measurements for this time (although this could also be related to the field of view). On the other hand, this modulation could be caused by an instability of lighter $H^{+}$ pick-up ions.

Alternatively, we have also to consider that it is also possible that these waves are not related to cometary ions; the overall structure is located not far from an HCS crossing occurring at the end of the day, and the small-scale perpendicular modulation of the field observed could be related to structures in the streamer. In any case, the large-scale dip in the magnetic field that contains the waves is quite remarkable and shares some similarities with the main structure studied in the rest of this work (e.g. the presence of some compression and number density increase in front of the structure, as in region D of Figure 3. The possibility cannot be excluded that this interval corresponds to the skimming of another cometary region shaped by the interaction with the solar wind, as earlier in the day.

\section{Discussion and comparison with other tail crossings}

Overall, the results presented here regarding both the largescale magnetic and plasma configuration and the small-scale properties of ion-scale waves provide us with quite strong evidence supporting the crossing by Solar Orbiter of a structure of cometary origin in the first part of 4 June. This is not too far from the expected date based on the revised impact parameter shown in Figure 1 and the time shift could be the consequence of an extended ion source from a trail of material along the comet's orbit.
It should also be noted that the suspected tail crossing took place well after the last ground-based observations of the comets' fragmented nucleus. As a result of fragmentation, the solar wind interaction region of comet ATLAS could be quite complex and its tail formed by different contributions from distinct comet fragments. In spite of this, we can reasonably assume that each element of the magnetotail would display the expected configuration with a more dense and low-field, slower plasma sheet surrounded by regions of anti-parallel field. This corresponds well to regions A-B-C in Figure 3

We can then compare this event with other tail crossings observed by other spacecraft. The configuration of the encounter here described displays some significant similarities with ICE observations at comet Giacobini-Zinner (GZ) (Smith et al. 1986, Bame et al. 1986). In particular, the GZ magnetotail configuration presented in Slavin et al. (1986) has a strong analogy to the reconstruction in Figure 3 . If the analogy holds, then regions A$\mathrm{B}-\mathrm{C}$ should be interpreted as the magnetotail plasma sheet surrounded by two lobes. Interestingly, the magnetic field intensity of the lobes seen in the GZ encounter was enhanced with respect the background solar wind, and Figure 3 suggests that this could be the case also for comet ATLAS. These observed signatures are also consistent with predictions of a tail crossing distant from the nucleus modelled by numerical simulations (Shou et al. 2015), namely, the flow deceleration and some large density modulation, including a density peak at the crossing of the central plasma sheet (low-field region B) and a big density dip in the surrounding lobes (region A and partially C). Moreover, these comparisons suggest that the two jumps in the magnetic field intensity, $B$ (highlighted by arrows in the top panel of Figure 3, associated with similar anti-correlated jumps in the density, could be the boundaries of the magnetotail. The second of them coincides with the sharp CS of Figure 4, located between regions C-D; since this separates the lobe with reversed polarity from the background IMF, it is accompanied by a large rotation also in the B-components. On the other hand, the first boundary, located at $\sim 23: 00$ on 3 June, at the beginning of region $A$, is embedded in plasma with same underlying magnetic field direction; consistent with this, the corresponding $B$ and $n$ jumps are not associated with strong rotations in the magnetic components.

Obviously, the timescale associated with the Solar Orbiter crossing is very different with respect to the GZ encounter. Beside differences in the size of the cometary environments and of the geometry of the s/c crossing in these cases, ICE approached the target comets much closer to their nucleus and the crossing of the tail only took a few minutes (though it spent a longer period in the cometosheath). Solar Orbiter approached the tail of comet ATLAS roughly a quarter of an AU downstream; the trajectory of Orbiter through the tail was a diagonal path through the tail, unlike the GZ tail crossing by ICE, which was essentially perpendicular to the Sun-Comet line. This is consistent with a more extended temporal crossing of the structure, as, for example, was the case with regard to the encounter of the comet Hyakutake by the Ulysses spacecraft (Jones et al.2000), although the latter was also a much more active comet, with a wider ion tail.

Another possible scenario is that the whole structure is a portion of the cometary tail that previously disconnected and was advected by the solar wind flow, embedded in the ambient plasma, namely, that a disconnection event had taken place (e.g. Vourlidas et al. 2007). This is schematically shown in the diagram of Figure 10 , similarly to the right panel of Figure 3 . In this case it is assumed that because the structure is advected by the solar wind - much faster than the s/c motion - the corresponding cut occurs approximately along the radial. This scenario re- 


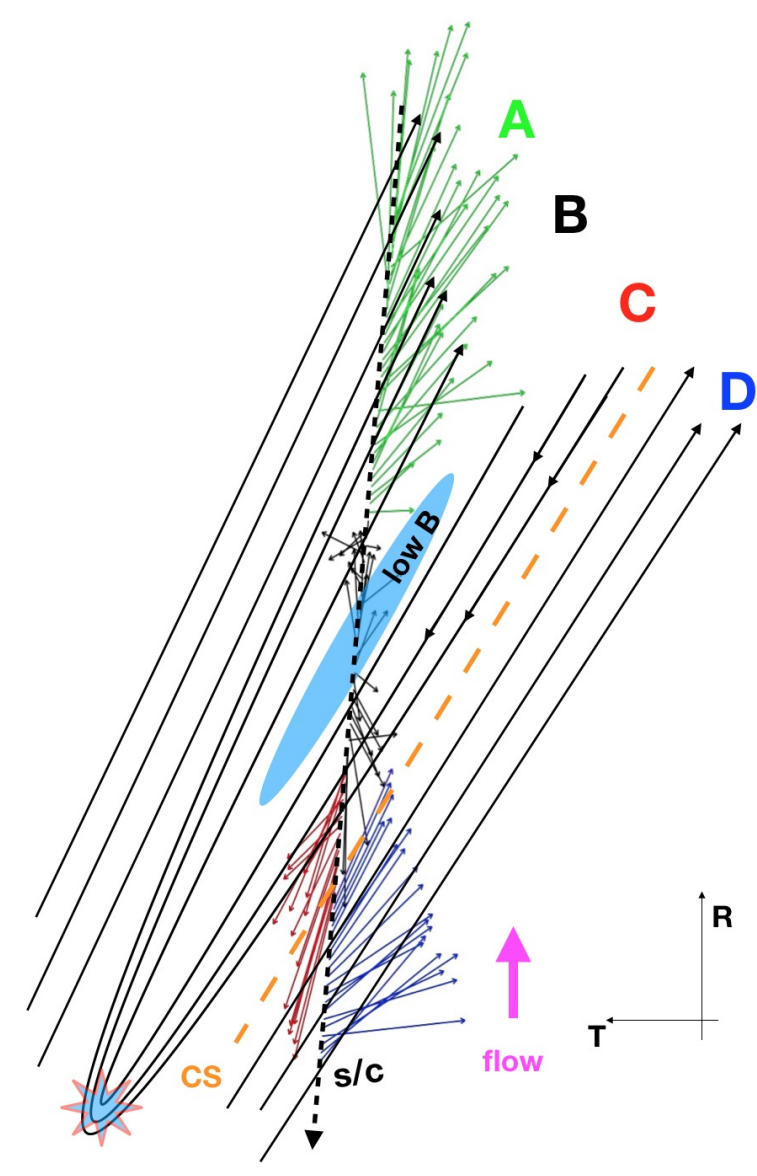

Fig. 10. Schematic reconstruction of the Solar Orbiter crossing in the case that the reverse field region corresponds to a cometary structure that is disconnected from the source and is advected by the solar wind. In this case, the crossing is not along the $\mathrm{s} / \mathrm{c}$ tangential motion as for a stationary structure, but corresponds to an approximately radial cut. The diagram has same notation as the cartoon in Figure 3 and magnetic field vectors are the same $B_{T}$ and $B_{R}$ projections as in panel $\mathrm{h}$ of the same figure. We note that in the case of a radial crossing, region $D$ is located upstream of the cometary obstacle

mains consistent with the main results discussed in the previous sections and, again, it supports the existence of distinct regions A-B-C-D around the draped field and the central low-field and the high-density, slower region $\mathrm{B}$. The only main difference with respect to the reconstruction of Figure 3, which is valid for a crossings mainly transverse with respect to the tail axis, is the relative position of the regions. From Figure 10, we may note that a radial crossing (solar wind advected structure) is consistent with region D lying in between the advected slower structure and the solar wind coming from below; in this configuration the increase in the number density flux observed in region D (panel e in Figure 3) could be interpreted as the compression of this region as the faster solar wind reaches the preceding cometary obstacle.

In order to assess, on a more quantitative level, the structure of the magnetotail, a more detailed analysis of the geometry of the crossing and, in particular, of the plasma sheet is needed. Even if this is probably difficult to accomplish due to the lack of plasma measurements in this interval, it could be the subject of future studies.

\section{Conclusions}

The period of the expected Solar Orbiter encounter with the tail of comet ATLAS has been considered analysing data from all in situ instruments. The original prediction by Jones et al. (2020) has been updated using the measured wind speed measured at the spacecraft and resulting in a minimum in the impact parameter around June 2-3 (Figure 1). Ashift to a later time is possible if, due to fragmentation, the source of cometary ions corresponds to an elongated region trailing behind the nominal nucleus position.

We have then identified on 4 June, the presence of a region with extremely low magnetic field, high plasma density, and lower solar wind speed surrounded by regions of oppositely directed magnetic field: one parallel and the other anti-parallel to the underlying IMF (Figure 3). The structure is not consistent with a polarity change due to a (double) crossing of the HCS and presents signatures of enhanced dust impacts and energetic ions. Inside the structure, the plasma appears to be in pressure balance - the large variations in $B$ are associated with equally large modulations of the density $n$ - and displays some strong velocity gradient as transitioning to the central low- $B$ region. We interpret this structure as the draped magnetic field of comet ATLAS ion tail and its central region as the tail plasma sheet. Overall the magnetic and plasma configuration observed by Solar Orbiter is analogous to that associated with a cometary induced magnetotail, as observed by previous spacecraft at different distances from the nucleus (Slavin et al. 1986, Jones et al. 2000) and consistent with MHD simulations (Shou et al. 2015). Moreover, the whole structure is bounded by two discontinuities in the magnetic field intensity and plasma density; the one sitting on the side of the magnetic lobe with reversed polarity corresponds to a sharp current sheet with a strong dip in $B$ due to the full rotation of the magnetic field components (Figure 4). This is consistent with a background interplanetary magnetic field direction that is close to radial during this interval, so that we expect a more asymmetric draping than for a transverse field; and this is also consistent with the asymmetric reconstruction of Figure 3 , that is, a smoother inversion on a side and sharper at the other.

In line with our interpretation, the whole interval of the structure and its surroundings are filled by an intense ion-scale waveactivity, with a variety of types of waveforms and structures. These include: trains of rotational discontinuities with little compression of $B$ (steepened Alfvén waves or KH vortices); transverse waves with either circular and linear polarization and associated with protons and heavier ions, respectively; and perpendicular modulation across the magnetic field, corresponding to pressure-balanced ion-scale filamentary structures aligned with the magnetic field. We interpret these features as generated by cometary pick-up ion instabilities and possibly by KH instability due to velocity shears at the internal boundaries of the structure. We identify, in particular, an interval with waves that are transverse with respect to the magnetic field but with linear polarization and whose instantaneous polarization axis rotates in time in the plane perpendicular to $\mathbf{B}$ with a period consistent with the $\mathrm{O}^{+}$ions gyroperiod (Figure 8 ). This matches the prediction from numerical simulations (Matteini et al. 2015) for an instability generated by cometary Oxygen pick-up ions with a initial nongyrotropic distribution (corresponding to unsteady source injection). Interestingly, these waves are detected outside the main region identified as the tail, but at a time when the heavy ion detector STEP displays the signature of another peak that is potentially related to the presence of heavy ions from the comet. This suggests that, as expected, cometary pick-up ions can be 
found also at some distance from the main source and influence the solar wind on a wider spatial region.

Moreover, other structures with similar properties - showing a decrease in $B$ and intense wave activity - are observed during the expected encounter period; one example is the field drop - associated with the $n$ increase - observed towards the end of June 4th, before the HCS crossing (Figure 9). The core of this structure is crossed by the $\mathrm{s} / \mathrm{c}$ at a large angle with respect to the (weak) magnetic field and reveals the presence of ion-scale, field-aligned, pressure balanced thin tubes with strong modulation across $\mathbf{B}$. The observed structure is potentially associated with a perpendicular instability (e.g. mirror or filamentation) triggered by ions with some large temperature anisotropy, such as pick-up ions, and we suggest that this could related to the presence of another cometary structure associated with a fragment of cometary ATLAS or to another disconnected tail piece advected by the solar wind. Unlike the main structure earlier in the day, this event does not display a field reversal (draping) and the dynamics of its detection could then correspond to the skimming of just a side of the magnetic structure by Solar Orbiter.

Finally, we note that this is the first comet tail crossing identified so close to the Sun $(0.5 \mathrm{AU})$ and it stands as one of the rare encounters with a comet that has gone through significant fragmentation (see also Gilbert et al. 2015). This and future possible encounters of this type in the inner heliosphere by Solar Orbiter and Parker Solar Probe constitute rather unique opportunities for the study of near-Sun comets and their interaction with the solar wind. The Solar Orbiter multi-instrumental and multi-scale first results presented in this study show once more the richness of the dynamics and demonstrate the feasibility of this type of investigation close to the Sun.

Acknowledgements. Solar Orbiter is a mission of international cooperation between ESA and NASA, operated by ESA. Solar Orbiter magnetometer operations are funded by the UK Space Agency (grant ST/T001062/1). TH and LW are supported by STFC grant ST/S000364/1, TW was supported by STFC grant ST/T506151/10 and RL by an Imperial College President's scholarship. JES is funded by the Royal Society University Research Fellowship URF/R1/201286. SRG and QA are supported by STFC PhD studentships. SDB acknowledges the support of the Leverhulme Trust Visiting Professor programme. The RPW instrument has been designed and funded by CNES, CNRS, the Paris Observatory, The Swedish National Space Agency, ESA-PRODEX and all the participating institutes. EPD/STEP was built at CAU and funded by the German Space Agency (DLR) under grant numbers 50OT0901, 50OT1202, 50OT1702 and 50OT2002. JRP and RGH acknowledge the financial support of the Spanish MICIU/AEI under projects ESP2017-88436-R and PID2019104863RBI00/AEI/10.13039/501100011033. Solar Orbiter Solar Wind Analyser (SWA) data are derived from scientific sensors which have been designed and created, and are operated under funding provided in numerous contracts from the UK Space Agency (UKSA), the UK Science and Technology Facilities Council (STFC), the Agenzia Spaziale Italiana (ASI), the Centre National d'Etudes Spatiales (CNES, France), the Centre National de la Recherche Scientifique (CNRS, France), the Czech contribution to the ESA PRODEX programme and NASA. Solar Orbiter SWA work at UCL/MSSL is currently funded under STFC grants ST/T001356/1 and ST/S000240/1.

\section{References}

Bame, S. J., Anderson, R. C., Asbridge, J. R., et al. 1986, Science, 232, 356

Borgogno, D., Hellinger, P., Passot, T., Sulem, P. L., \& Trávníček, P. M. 2009, Nonlinear Processes in Geophysics, 16, 275

Califano, F., Hellinger, P., Kuznetsov, E., et al. 2008, Journal of Geophysical Research (Space Physics), 113, A08219

Coates, A. J. 2004, Advances in Space Research, 33, 1977

Combi, M. R., Shou, Y., Mäkinen, T., et al. 2021, Icarus, 365, 114509

Delva, M., Zhang, T. L., Volwerk, M., et al. 2008, Geophysical Research Letters, 35

Finson, M. J. \& Probstein, R. F. 1968, ApJ, 154, 327

Gilbert, J. A., Lepri, S. T., Rubin, M., Combi, M., \& Zurbuchen, T. H. 2015, ApJ, 815,12
Gloeckler, G., Allegrini, F., Elliott, H. A., et al. 2004, ApJ, 604, L121

Horbury, T. S., O’Brien, H., Carrasco Blazquez, I., et al. 2020, A\&A, 642, A9

Ip, W.-H. 2004, Global solar wind interaction and ionospheric dynamics, ed. M. C. Festou, H. U. Keller, \& H. A. Weaver, 605-629

Jian, L. K., Russell, C. T., Luhmann, J. G., et al. 2010, Journal of Geophysical Research (Space Physics), 115, A12115

Jian, L. K., Wei, H. Y., Russell, C. T., et al. 2014, ApJ, 786, 123

Jones, G. H., Afghan, Q., \& Price, O. 2020, Research Notes of the American Astronomical Society, 4, 62

Jones, G. H., Balogh, A., \& Horbury, T. S. 2000, Nature, 404, 574

Jones, G. H., Forsyth, R. J., \& Coates, A. J. 2010, in American Institute of Physics Conference Series, Vol. 1302, Pickup Ions Throughout the Heliosphere and Beyond, ed. J. Le Roux, G. P. Zank, A. J. Coates, \& V. Florinski, 225-230

Jones, G. H., Knight, M. M., Battams, K., et al. 2018, Space Sci. Rev., 214, 20

Kasper, J. C. 2019, 825, L26

Khotyaintsev, Y. V., Graham, D. B., Vaivads, A., et al. 2021, arXiv e-prints, arXiv:2103.17208

Kieokaew, R., Lavraud, B., Yang, Y., et al. 2021, arXiv e-prints, arXiv:2103.15489

Laker, R., Horbury, T. S., Bale, S. D., et al. 2021, arXiv e-prints, arXiv:2103.00230

Laveder, D., Passot, T., \& Sulem, P. L. 2002, Physics of Plasmas, 9, 293

Maksimovic, M., Bale, S. D., Chust, T., et al. 2020, A\&A, 642, A12

Matteini, L., Franci, L., Alexandrova, O., et al. 2020, Frontiers in Astronomy and Space Sciences, 7, 83

Matteini, L., Schwartz, S. J., \& Hellinger, P. 2015, 119, 3

Mazelle, C. \& Neubauer, F. M. 1993, Geophys. Res. Lett., 20, 153

Müller, D., St. Cyr, O. C., Zouganelis, I., et al. 2020, A\&A, 642, A1

Neugebauer, M., Gloeckler, G., Gosling, J. T., et al. 2007, ApJ, 667, 1262

Neugebauer, M., Lazarus, A. J., Balsiger, H., et al. 1989, 94, 5227

Owen, C. J., Bruno, R., Livi, S., et al. 2020, A\&A, 642, A16

Píša, D., Souček, J., Santolík, O., et al. 2021, arXiv e-prints, arXiv:2104.03082

Rodríguez-Pacheco, J., Wimmer-Schweingruber, R. F., Mason, G. M., et al. 2020, A\&A, 642, A7

Shou, Y., Combi, M., Jia, Y. D., et al. 2015, ApJ, 809, 156

Shukla, P. K. \& Stenflo, L. 1989, Physics of Fluids B, 1, 1926

Slavin, J. A., Smith, E. J., Tsurutani, B. T., et al. 1986, Geophys. Res. Lett., 13, 283

Smith, E. J., Tsurutani, B. T., Slavin, J. A., et al. 1986, Science, 232, 382

Soucek, J., Lucek, E., \& Dandouras, I. 2008, Journal of Geophysical Research (Space Physics), 113, A04203

Stansby, D., Horbury, T. S., \& Matteini, L. 2019, 482, 1706

Steinvall, K., Khotyaintsev, Y. V., Cozzani, G., et al. 2021, arXiv e-prints, arXiv:2104.03553

Szegö, K., Glassmeier, K.-H., Bingham, R., et al. 2000, Space Sci. Rev., 94, 429

Tsurutani, B. T., Lakhina, G. S., Sen, A., et al. 2018, Journal of Geophysical Research (Space Physics), 123, 2458

Tsurutani, B. T. \& Smith, E. J. 1986, 13, 263

Verniero, J. L., Larson, D. E., Livi, R., et al. 2020, ApJS, 248, 5

Vourlidas, A., Davis, C. J., Eyles, C. J., et al. 2007, ApJ, 668, L79

Wang, Y.-M. 2010, 715, L121

Wimmer-Schweingruber, R., Janitzek, N., Pacheco, D., et al. 2021, Astron.\& Astrophys., manuscript submitted

Woodham, L. D., Wicks, R. T., Verscharen, D., \& Owen, C. J. 2018, ApJ, 856, 49

Woodham, L. D., Wicks, R. T., Verscharen, D., et al. 2019, ApJ, 884, L53

Zouganelis, I., De Groof, A., Walsh, A. P., et al. 2020, A\&A, 642, A3 
1 Department of Physics, Imperial College London, South Kensington Campus, London SW7 2AZ, UK

e-mail: 1.matteini@imperial.ac.uk

2 Physics Department, University of California, Berkeley, CA 947207300, USA

3 Space Sciences Laboratory, University of California, Berkeley, CA 94720-7450, USA

${ }^{4}$ Swedish Institute of Space Physics (IRF), Uppsala, Sweden

5 Space and Plasma Physics, Department of Physics and Astronomy, Uppsala University, Uppsala 75120, Sweden

6 UCL Mullard Space Science Laboratory, Holmbury St. Mary, Dorking RH5 6NT, UK

7 The Centre for Planetary Sciences at UCL/Birkbeck, Gower Street, London WC1E 6BT, UK

${ }^{8}$ LESIA, Observatoire de Paris, Université PSL, CNRS, Sorbonne Université, Univ. Paris Diderot, Sorbonne Paris Cité, 5 place Jules Janssen, 92195 Meudon, France

9 LPP, CNRS, Ecole Polytechnique, Sorbonne Université, Observatoire de Paris, Université Paris-Saclay, Palaiseau, Paris, France

10 LPC2E, CNRS, 3A avenue de la Recherche Scientifique, Orléans, France

11 Université d'Orléans, Orléans, France

12 CNES, 18 Avenue Edouard Belin, 31400 Toulouse, France

13 Technische Universitat Dresden, Wurzburger Str. 35, D-01187 Dresden, Germany

14 Institute of Atmospheric Physics of the Czech Academy of Sciences, Prague, Czechia

15 Space Research Institute, Austrian Academy of Sciences, Graz, Austria

16 Astronomical Institute of the Czech Academy of Sciences, Prague, Czechia

17 Department of Space and Plasma Physics, School of Electrical Engineering and Computer

18 Radboud Radio Lab, Department of Astrophysics, Radboud University, Nijmegen, The Netherlands

19 Institut für Experimentelle und Angewande Physik, ChristianAlbrechts-Universität zu Kiel, 24118 Kiel, Germany

20 Johns Hopkins University, Applied Physics Laboratory

21 Universidad de Alcalá, Dpto. de Física y Matemáticas, Space Research Group, 28805, Alcalá de Henares, Spain

22 Institut de Recherche en Astrophysique et Planétologie, CNRS, Université de Toulouse, CNES, Toulouse, France.

23 INAF-Istituto di Astrofisica e Planetologia Spaziali, Via Fosso del Cavaliere 100, 00133 Roma, Italy.

24 Southwest Research Institute, 6220 Culebra Road, San Antonio TX, 78238, USA.

25 European Space Agency, ESAC, Camino Bajo del Castillo s/n, Urb. Villafranca del Castillo, 28692 Villanueva de la Canada, Madrid, Spain

26 European Space Agency, ESTEC, PO Box 299, 2200 AG Noordwijk, The Netherlands 